\title{
A novel completed LPV controller and observer scheme in order to control nonlinear compartmental systems
}

\author{
György Eigner*, Péter Pausits ${ }^{\dagger}$ and Levente Kovács* \\ * Research and Innovation Center of Óbuda University, \\ Physiological Controls Group, Óbuda University, Budapest, Hungary \\ Email: \{eigner.gyorgy,kovacs.levente\} @ nik.uni-obuda.hu \\ ${ }^{\dagger}$ Research and Innovation Center of Óbuda University, \\ Antal Bejczy Center for Intelligent Robotics, Óbuda University, Budapest, Hungary \\ Email: peter.pausits@irob.uni-obuda.hu
}

\begin{abstract}
The purpose of this study is to introduce a novel "completed" LPV controller and observer design approach for control of particular class of nonlinear systems. The developed tools allow to use classical linear controller design theorems via LPV framework without the advanced Linear Matrix Inequality (LMI) theorems. The developed method combines the classical state feedback theorems and a supplementary controller and observer structure which basis is the special properties of the parameter space of the linear parameter varying (LPV) system. The main benefit of the proposed method is that the controller design does not require highly advanced mathematical tools and high computational capacity. We have proven the usability of the method in case of a highly nonlinear compartmental model. The results have shown that the completed LPV controller is able to handle the system with good performance.
\end{abstract}

Index terms - Linear Parameter Varying, Nonlinear Systems, Compartmental Models,

\section{INTRODUCTION}

Most of the physiological systems in the real world have nonlinear behavior. In order to control them nonlinear controller design approaches or linear controller design solutions can be used - however, the latter frameworks only can control the real nonlinear systems in a particular operation domain [1].

A possible choice to deal with nonlinear control by itself is the usage of Lyapunov's methods [2], [3]. Lyapunov's work provided a universal mathematical tool which let the researchers to decide whether a nonlinear system is stable or not and based on that the nonlinear controller design is possible. Lyapunov's second or direct method provides a way to determine the stability of a nonlinear system without solving the equations of motion. Due to the fact that most of the real life problems do not have analytical solutions in closed form and the validity of numerical solutions are limited, Lyapunov's method is extremely useful and most of the controller design methods are based on that even today [2], [3].
In the last decade, several solutions were appeared based on Lyapunov's framework which purpose to develop generalized solutions for nonlinear control. However, most of them are requires the highly creative thinking of an expert designer, high computational capacity and advanced mathematical solutions (eg. LMI) based optimizations.

Other solutions are exists, for example, the Robust Fixed Point Transformation (RFPT)-based controller design [4] framework, which only consider an approximation of the real system. The method is able to provide robust and adaptive controller for nonlinear systems by using the fix point theorems and contractive Cauchy sequences in the Banach space [5].

An other solution is the LPV methodology. The main benefit of the LPV framework is it does allow the usage of linear controller design approaches by "hiding" the nonlinearities of given systems [6]. However, most of the cases, the LPV methods are combined with Lyapunov's theorems via LMI framework.

In this study we demonstrated an alternative and novel controller design approach, which exploits the mathematical properties of the abstract parameter space of the LPV systems and the matrix similarity theorems [7], [8].

The paper structured, as follows: first, we introduce the basics of LPV systems; then, we present the novel LPV controller and observer scheme and the ideas behind; afterwards, we demonstrate the developed method on given nonlinear compartmental model; finally, we conclude our work and present the future directions.

\section{LPV SYSTEM DESCRIPTION IN STATE SPACE FORM}

In the followings we defined the necessary definitions which are required to describe the LPV systems.

Definition 1. Scheduling variable or parameter: real valued scalar or function, which is a multiplied out term of a mathematical model and determines a particular property of the model. Notations: scalar case: $p=\left\{p \in \mathbb{R}, p_{\text {min }} \leq p \leq\right.$ $\left.p_{\max }\right\}$; function case: $p(x)=\left\{p(x) \in \mathbb{R}, p_{\min } \leq p(x) \leq\right.$ $\left.p_{\max }\right\}$. 
Remark 1.1. In this study we focused to those cases, when the scheduling parameter is a function of time: $p(t)=\{p(t) \in$ $\left.\mathbb{R}, p_{\min } \leq p(t) \leq p_{\max }\right\}$

Remark 1.2. The scheduling variables can be complex valued scalars or functions and they may depend on the properties of the mathematical models too. This Theses does not discuss this possibility, since the investigated systems do not have such properties.

The purpose of the selection of the scheduling variables are manyfold. If, the scheduling variables are simple parameters, the LPV model becomes appropriate to describe multiple model cases via the varying of the parameters. A more sophisticated reason is to select those functions (mostly time functions) as scheduling variables which cause nonlinearities in order to avoid this unfavorable property.

Definition 2. Parameter vector: a real valued, bounded permanent or time varying vector, which consist of scheduling variables. Notations: permanent case: $\mathbf{p}$; time varying case: $\mathbf{p}(t)$. The dimension of the parameter vector is equal to the number of selected scheduling variables, namely, $\mathbf{p}(t) \in \mathbb{R}^{q}$.

The literature distinguishes between the LPV models according to the fact whether the selected scheduling variables are not state variables (LPV) or they are state variables also selected as scheduling parameters (qLPV). Nevertheless, there is no difference between them from notation point of view. However, the eligible interpretation of the cases is important to be noticed.

Definition 3. Parameter Space (PS): a $q$ dimensional real vector space $\mathbb{R}^{q}$, where each dimension represents the possible values of a given scheduling variable.

Definition 4. Parameter box (PB): a $q$ dimensional simplex inside the $\mathbb{R}^{q}$, which is determined by the minimum $p_{i, \min }$ and maximum $p_{i, \max }$ values of the scheduling variables $p_{i}(t)$. Usually, the PB represents those space which is the meaningful region of the parameters from the physical or physiological point of view. The size of the PB (the minimum and maximum values of the scheduling variables) can be tighter as that allowed by the reality - in this case the PB means the investigated region.

The general state space (SS) representation of LPV systems, where disturbance is not considered can be described as follows:

$$
\begin{aligned}
& \dot{\mathbf{x}}(t)=\mathbf{A}(\mathbf{p}(t)) \mathbf{x}(t)+\mathbf{B}(\mathbf{p}(t)) \mathbf{u}(t) \\
& \mathbf{y}(t)=\mathbf{C}(\mathbf{p}(t)) \mathbf{x}(t)+\mathbf{D}(\mathbf{p}(t)) \mathbf{u}(t)
\end{aligned}
$$

where $\mathbf{x}(t) \in \mathbb{R}^{n}$ is the state vector, $\mathbf{y}(t) \in \mathbb{R}^{k}$ is the output vector, $\mathbf{u}(t) \in \mathbb{R}^{m}$ is the output vector, $\mathbf{A}(\mathbf{p}(t)) \in \mathbb{R}^{n \times n}$ is the state matrix, $\mathbf{B}(\mathbf{p}(t)) \in \mathbb{R}^{n \times m}$ is the control input matrix, $\mathbf{C}(\mathbf{p}(t)) \in \mathbb{R}^{k \times n}$ is the output matrix and $\mathbf{D}(\mathbf{p}(t)) \in \mathbb{R}^{k \times m}$ is the control feed-forward matrix.

The matrices of (1) can be unified:

$$
\mathbf{S}(\mathbf{p}(t))=\left(\begin{array}{ll}
\mathbf{A}(\mathbf{p}(t)) & \mathbf{B}(\mathbf{p}(t)) \\
\mathbf{C}(\mathbf{p}(t)) & \mathbf{D}(\mathbf{p}(t))
\end{array}\right)
$$

where $\mathbf{S}(\mathbf{p}(t)) \in \mathbb{R}^{(n+k) \times(n+m)}$ is the system matrix.

The compact form of general LPV system from (1) becomes:

$$
\left(\begin{array}{c}
\dot{\mathbf{x}}(t) \\
\mathbf{y}(t)
\end{array}\right)=\mathbf{S}(\mathbf{p}(t))\left(\begin{array}{c}
\mathbf{x}(t) \\
\mathbf{u}(t)
\end{array}\right) .
$$

The classical approaches that use LPV form in modeling apply general, affine and polytopic LPV system models [6], [9], [10]. In this study we have used the affine LPV theorem to describe a given nonlinear system.

In this case affine functions of the parameter vector are used to describe the LPV systems. The affine LPV system in SS form consists of two parts: (i) Permanent part: this term is independent from the $\mathbf{p}(t)$ and (ii) Varying part: this term is dependent from the $\mathbf{p}(t)$. The system matrix in affine case is the following:

$$
\mathbf{S}(\mathbf{p}(t))=\left(\begin{array}{ll}
\mathbf{A}_{0}+\sum_{i=1}^{q} p_{i}(t) \mathbf{A}_{i} & \mathbf{B}_{0}+\sum_{i=1}^{q} p_{i}(t) \mathbf{B}_{i} \\
\mathbf{C}_{0}+\sum_{i=1}^{q} p_{i}(t) \mathbf{C}_{i} & \mathbf{D}_{0}+\sum_{i=1}^{q} p_{i}(t) \mathbf{D}_{i}
\end{array}\right),
$$

where $\mathbf{A}_{0}, \mathbf{B}_{0}, \mathbf{C}_{0}$ and $\mathbf{D}_{0}$ are permanent and they are independent from the parameter vector.

In affine case the $\mathbf{S}(\mathbf{p}(t))$ in (3) is equal to (4). The affine LPV models keep their validity only in inside the PB during operation.

\section{IDEAS BEHIND THE NOVEL LPV CONTROLLER AND OBSERVER SCHEME}

\section{A. Classical State Feedback Controller and Observer Design}

A Linear Time Invariant (LTI) system can be described by $\mathbf{A}, \mathbf{B}, \mathbf{C}$ and $\mathbf{D}$ matrices in SS form:

$$
\left(\begin{array}{l}
\dot{\mathbf{x}}(t) \\
\mathbf{y}(t)
\end{array}\right)=\left[\begin{array}{ll}
\mathbf{A} & \mathbf{B} \\
\mathbf{C} & \mathbf{D}
\end{array}\right]\left(\begin{array}{l}
\mathbf{x}(t) \\
\mathbf{u}(t)
\end{array}\right)=\mathbf{S}(\mathbf{p}(t))\left(\begin{array}{l}
\mathbf{x}(t) \\
\mathbf{u}(t)
\end{array}\right) .
$$

In case of state feedback control, the control signal occurs in the following form:

$$
\mathbf{u}(t)=-\mathbf{K} \mathbf{x}(t),
$$

where the control input $\mathbf{u}(t)$ is the linear combination of the feedback gain matrix $\mathbf{K} \in \mathbb{R}^{m \times n}$ and the state vector $\mathbf{x}(t)$. The $\mathbf{K}$ can be designed via different iteration-based methods, for example, Linear-Quadratic (LQ) control [11]. The optimal $\mathbf{K}$ gain ensures that LTI systems which are non-stable or stable but do not have eligible properties become stable, with better control performances through pole-placement. In general, this configuration modifies the open-loop $\mathbf{A}_{\text {open }}$ state matrix into $\mathbf{A}_{\text {closed }}=\mathbf{A}_{\text {open }}-\mathbf{B K}$. The poles of the characteristic equation can be calculated as follows: 


$$
|\mathbf{I} \lambda-\mathbf{A}+\mathbf{B K}|=0
$$

and the closed loop poles $\lambda_{\text {closed }}$ fulfill the requirements of stability [11], [12].

In case of full order linear observer for LTI systems, the linear observer is a dynamic system which output is the $\hat{\mathbf{x}}(t)$ estimated state vector. If, the observer is asymptotic, the estimation error, namely $\tilde{\mathbf{x}}(t):=\mathbf{x}(t)-\hat{\mathbf{x}}(t)$ has to converge to zero over time [13].

The general form of the full order linear observer can be described as follows:

$$
\dot{\hat{\mathbf{x}}}(t)=\mathbf{F} \hat{\mathbf{x}}(t)+\mathbf{G y}(t)+\mathbf{H u}(t)
$$

where $\mathbf{F} \in \mathbb{R}^{n \times n}$ is the observer state matrix, $\mathbf{G} \in \mathbb{R}^{k \times n}$ is the observer gain matrix and $\mathbf{H} \in \mathbb{R}^{n \times m}$ is the observer input matrix.

The velocity of the disappearance of the observation error can be prescribed by the eigenvalues of the $\mathbf{F}$, which is traceable to the determination of the characteristic polynomial of $\mathbf{F}$ [11], [13]:

$$
|s \mathbf{I}-\mathbf{F}|=|s \mathbf{I}-\mathbf{A}-\mathbf{G} \mathbf{C}|=\left|s \mathbf{I}-\mathbf{A}^{T}-\mathbf{C}^{T} \mathbf{G}^{T}\right| .
$$

In this way an asymptotic state observer design leads to a state feedback design task, where the $\mathbf{G}$ observer gain provide that the closed loop poles of the observer becomes to equal to the predefined observer poles.

\section{B. Mathematical Tools and Ideas}

In the PS, the $\mathbf{p}(t)$ uniquely determines the underlying belonging $\mathbf{S}(\mathbf{p}(t))$ [7]. If, the $\mathbf{p}$ is fixed $\mathbf{S}(\mathbf{p}(t))$ simplified to an underlying LTI system $\mathbf{S}$.

Definition 5. Norm based difference in the PS among LTI systems: The difference between every occurring LTI systems $\mathbf{S}_{i}, \mathbf{S}_{j}$ in the PS can be characterized by the $\mathcal{L}_{2}$ norm of the difference between the belonging parameter vectors: $e:=\left\|\mathbf{p}_{i}-\mathbf{p}_{j}\right\|_{2}[7]$.

Remark 5.1. By using the consequences of Definition 5, it is possible to describe the dissimilarity of two underlying LTI systems in the PS. Moreover, it can be used to describe the difference between a fixed LTI system $\mathbf{S}_{f i x}$ and a varying one $\mathbf{S}_{\text {var }}(t)$ over time through they belonging parameter vectors: $e(t)=\left\|\mathbf{p}_{\text {fix }}-\mathbf{p}_{\text {var }}(t)\right\|_{2}[7]$.

Definition 6. Similarity of matrices: A quadratic, $n \times n$ matrix $\mathbf{M}$ is similar to a matrix $\mathbf{T}$, if it is exist an invertible $\mathbf{Z}$ matrix that is $\mathbf{M}=\mathbf{Z}^{-1} \mathbf{T Z}$. Notation: $\mathbf{M} \sim \mathbf{T}[8]$.

This definition has wide ranging applications. Two of them are the following theorems, whose proof can be found in various sources, among others, in [8], [14]:

Theorem 1. Similarity invariance of the determinants of matrices: If $\mathbf{M} \sim \mathbf{T}$, then $|\mathbf{M}|=|\mathbf{T}|$.
Proof 1.1. Let $\mathbf{M} \sim \mathbf{T}$, namely, $\mathbf{M}=\mathbf{Z}^{-1} \mathbf{T Z}$. Then $|\mathbf{M}|=\left|\mathbf{Z}^{-1} \mathbf{T Z}\right|=\left|\mathbf{Z}^{-1}\right||\mathbf{T}||\mathbf{Z}|=|\mathbf{T}|$, since $|\mathbf{Z}|\left|\mathbf{Z}^{-1}\right|=1$ [8].

Theorem 2. If $\mathbf{M} \sim \mathbf{T}$, then the characteristic polynomials of the matrices and thus, the eigenvalues and the geometric and algebraic multiplicities of the eigenvalues of the matrices are the same.

Proof 2.1. Let $\mathbf{M} \sim \mathbf{T}$, namely, $\mathbf{M}=\mathbf{Z}^{-1} \mathbf{T Z}$. Then $\mathbf{M}-\lambda \mathbf{I}=\mathbf{Z}^{-1} \mathbf{T Z}-\lambda \mathbf{Z}^{-1} \mathbf{I Z}=\mathbf{Z}^{-1}(\mathbf{T Z}-\lambda \mathbf{I Z})=$ $\mathbf{Z}^{-1}(\mathbf{T}-\lambda \mathbf{I}) \mathbf{Z}$, namely, $\mathbf{M}-\lambda \mathbf{I} \sim \mathbf{T}-\lambda \mathbf{I}$ [8].

These mathematical tools can be used to define eigenvalues equality rules for state feedback systems and allows us to complete the state feedback structures.

\section{General properties of physiological systems}

Generally, the following properties are true in case of physiological systems, especially, regarding to compartmental models [1]:

- Input(s) are not affected by nonlinearities and do not have direct connection between the inputs and outputs (the consequence being that $\mathbf{D}=\mathbf{0}$ and it is persistent in time);

- Output(s) are not affected by nonlinearities;

- Since the nonlinearities do not affect the inputs and the outputs, it is not necessary to select their elements as scheduling parameters, which means that $\mathbf{B}$ and $\mathbf{C}$ are independent from the parameter vector $\mathbf{p}$; moreover, these usually do not depend on time;

- The nonlinearities only appear in the state matrix $\mathbf{A}(\mathbf{p}(t))$ regarding to the nonlinear system dynamics, nonlinear cross effects and nonlinear coupling; the patient variabilities are mostly occur in the elements of $\mathbf{A}$.

\section{Completed Controller and Observer Design and Scheme}

1) Completed Controller Gain: In the PS, each point describes a LTI system $\mathbf{S}\left(\mathbf{p}_{i}\right)$ and its belonging parameter vector is $\mathbf{p}_{i}$. It is possible to design a state feedback based optimal or robust controller, where the feedback gain $\mathbf{K}\left(\mathbf{p}_{i}\right)$ is able to handle the LTI system and provide stability, good performance, etc.

Consider a reference LTI system $\mathbf{S}\left(\mathbf{p}_{\text {ref }}\right)$ and the actual LPV system $\mathbf{S}(\mathbf{p}(t))$, which varies over time. The 2-norm based difference $e(t)$ can be used to describe the difference between them through they belonging parameter vectors $e(t):=\left\|\mathbf{p}_{\text {ref }}-\mathbf{p}(t)\right\|_{2}$. From the PS point of view, the dissimilarity of the $\mathbf{S}_{r e f}$ and $\mathbf{S}(\mathbf{p}(t))$ can be described by $e(t)$.

The key aspect is that the previously defined Theorem 1 and Theorem 2 allow to introduce such a completed controller and observer scheme, which prescribed that the eigenvalues of the closed LPV system have to be equal to the closed LTI system.

Consider that $\mathbf{A}_{\text {ref }}-\mathbf{B} \mathbf{K}_{\text {ref }} \sim \mathbf{A}(\mathbf{p}(t))-\mathbf{B}\left(\mathbf{K}_{r e f}+\right.$ $\mathbf{K}(t) e(t))$, which means that the eigenvalues of the closed loop reference matrix $\lambda\left(\mathbf{p}_{\text {ref }}\right)$ and the closed loop varying 
parameter dependent matrix $\lambda(\mathbf{p}(t))$ become to equal during operation. Namely, $\lambda\left(\mathbf{p}_{\text {ref }}\right)=\lambda(\mathbf{p}(t))$ at $\forall \mathbf{p}(t)$, if $\lambda(\mathbf{p}(t))$ is the eigenvalues of $\left(\mathbf{A}(\mathbf{p}(t))-\mathbf{B}\left(\mathbf{K}_{r e f}+\mathbf{K e}(t)\right)\right)$. This is only possible, if the similarity transformation matrix is the $\mathbf{I}_{n \times n}$ unity matrix. Hence, $\mathbf{A}_{r e f}-\mathbf{B K}_{r e f}=\mathbf{I}^{-1}(\mathbf{A}(\mathbf{p}(t))-$ $\left.\mathbf{B}\left(\mathbf{K}_{r e f}+\mathbf{K}(t) e(t)\right)\right) \mathbf{I}$, i.e. the introduced completed gain has to provide the "smoother" similarity, but also the "strict" equality criteria. Shortly, the proposed completed feedback gain $\mathbf{K}_{r e f}+\mathbf{K}(t) e(t)$ has to provide the equality of not just the eigenvalues $\lambda\left(\mathbf{p}_{\text {ref }}\right)=\lambda(\mathbf{p}(t))$, but also the equality of the matrices, as well:

$$
\mathbf{A}_{r e f}-\mathbf{B} \mathbf{K}_{r e f}=\mathbf{A}(\mathbf{p}(t))-\mathbf{B}\left(\mathbf{K}_{r e f}+\mathbf{K}(t) e(t)\right) .
$$

This key aspect let us to define the completed control gain $\mathbf{K}(t)$ [15], [16].

In that manner, the closed-loop LPV system with state feedback controller becomes:

$$
\mathbf{A}(\mathbf{p}(t))-\mathbf{B}\left(\mathbf{K}_{r e f}+\mathbf{K}(t) e(t)\right),
$$

where $\mathbf{K}(t)_{m \times n}$ is a continuously calculable gain. That means, that $\mathbf{K}(t)$ can be calculated for every occurring LTI system via $e(t)$ :

$$
\begin{aligned}
\mathbf{K}(t) & =\frac{\mathbf{B}^{-1}\left(\mathbf{A}_{r e f}-\mathbf{B K}_{r e f}-\mathbf{A}(\mathbf{p}(t))+\mathbf{B K}_{r e f}\right)}{-e(t)} \\
\mathbf{K}(t) & =\frac{\mathbf{B}^{-1}\left(\mathbf{A}_{r e f}-\mathbf{A}(\mathbf{p}(t))\right.}{-e(t)}
\end{aligned}
$$

2) Completed Observer Gain: Theorems 1 and 2 can be used similar to (11) and (12) to define the completed observer gain $\mathbf{G}(t)$.

Let $\mathbf{F}=\mathbf{A}_{r e f}-\mathbf{G}_{r e f} \mathbf{C} \sim \mathbf{F}(t)=\mathbf{A}(\mathbf{p}(t))-\left(\mathbf{G}_{r e f}+\right.$ $\mathbf{G}(t) e(t)) \mathbf{C}$, which means that the eigenvalues of $\mathbf{F} \lambda(\mathbf{F})$ and $\lambda(\mathbf{F}(t))$ become to equal during operation. So, $\lambda(\mathbf{F})=$ $\lambda(\mathbf{F}(t))$ at $\forall \mathbf{p}(t)$, if $\lambda(\mathbf{F}(t))$ is the eigenvalues of $\mathbf{F}(t)=$ $\mathbf{A}(\mathbf{p}(t))-\left(\mathbf{G}_{r e f}+\mathbf{G}(t) e(t)\right) \mathbf{C}$. This is only possible, if the similarity transformation matrix is the $\mathbf{I}_{n \times n}$ unity matrix. Namely, $\mathbf{F}=\mathbf{I}^{-1} \mathbf{F}(t) \mathbf{I}$. As previously, that means that the introduced observer gain has to provide the "smoother" similarity, but also the "strict" equality criteria. Shortly, the proposed completed observer gain $\mathbf{G}_{r e f}+\mathbf{G}(t) e(t)$ has to provide the equality of not just the eigenvalues $\lambda(\mathbf{F})=\lambda(\mathbf{F}(t))$, but also the equality of the matrices, as well:

$$
\begin{aligned}
& \mathbf{F}=\mathbf{F}(t) \\
& \mathbf{A}_{r e f}-\mathbf{G}_{r e f} \mathbf{C}=\mathbf{A}(\mathbf{p}(t))-\left(\mathbf{G}_{r e f}+\mathbf{G}(t) e(t)\right) \mathbf{C}
\end{aligned}
$$

Hence, (13) can be rearranged to calculate $\mathbf{G}(t)$ :

$$
\begin{aligned}
& \begin{array}{l}
\mathbf{A}_{r e f}-\mathbf{G}_{r e f} \mathbf{C}=\mathbf{A}(\mathbf{p}(t))-\mathbf{G}_{r e f} \mathbf{C}-\mathbf{G}(t) \mathbf{C} e(t) \\
\left(\mathbf{A}_{r e f}-\mathbf{G}_{r e f} \mathbf{C}-\mathbf{A}(\mathbf{p}(t))+\mathbf{G}_{r e f} \mathbf{C}\right) \mathbf{C}^{-1} \\
=-\mathbf{G}(t) \mathbf{C C}^{-1} e(t) \\
\mathbf{G}(t)=\frac{\left(\mathbf{A}_{r e f}-\mathbf{G}_{r e f} \mathbf{C}-\mathbf{A}(\mathbf{p}(t))+\mathbf{G}_{r e f} \mathbf{C}\right) \mathbf{C}^{-1}}{-e(t)} . \\
\mathbf{G}(t)=\frac{\left(\mathbf{A}_{r e f}-\mathbf{A}(\mathbf{p}(t))\right) \mathbf{C}^{-1}}{-e(t)}
\end{array}
\end{aligned}
$$

3) Completed controller scheme: We did not list here the consequences, limitations and usability of the continuously calculable controller $\mathbf{K}(t)$ and observer $\mathbf{G}(t)$ gains (13) and (14) due to the lack of space. The main limitation are connected to the invertibility questions of $\mathbf{B}$ and $\mathbf{C}$; the structure of the system matrix $\mathbf{S}(\mathbf{p}(t))$; and to the singularity of $\mathbf{K}(t)$ and $\mathbf{G}(t)$. The deep investigation of these remarkable questions can be found in [15], [16].

Here we limit ourselves to provide the solution to avoid the singularity of $\mathbf{K}(t)$ and $\mathbf{G}(t)$. When $e(t)=0$, the $\mathbf{K}(t)$ and $\mathbf{G}(t)$ become singular. This can be easily avoided, if we consider the usage of a strict bound in the small environment of $e(t)=0$ :

$$
\mathbf{K}(t)=\left\{\begin{array}{ll}
\mathbf{0} & \text { if }-\epsilon<e(t)<\epsilon \\
\frac{\mathbf{B}^{-1}\left(\mathbf{A}_{r e f}-\mathbf{A}(\mathbf{p}(t))\right.}{-e(t)} & \text { otherwise }
\end{array},\right.
$$

$$
\mathbf{G}(t)= \begin{cases}0 & \text { if }-\epsilon<e(t)<\epsilon \\ \frac{\left(\mathbf{A}_{r e f}-\mathbf{A}(\mathbf{p}(t))\right) \mathbf{C}^{-1}}{-e(t)} & \text { otherwise }\end{cases}
$$

where $\epsilon$ is a real number and determines a small environment around the $\mathbf{p}_{\text {ref }}$ in the PS. That means, if the $e(t)$ nears to zero, the $\mathbf{K}(t)=\mathbf{0}$ and $\mathbf{G}(t)=\mathbf{0}$ and only $\mathbf{K}_{r e f}$ and $\mathbf{G}_{r e f}$ are used from (11) an (13). Hence $e(t)=0$ denotes that $\mathbf{p}_{\text {ref }}=\mathbf{p}(t)$ and $\mathbf{S}\left(\mathbf{p}_{\text {ref }}\right)=\mathbf{S}(\mathbf{p}(t))$. That is, those LTI system, which occurs, when $\mathbf{p}(t)$ nears to $\mathbf{p}_{\text {ref }}$, namely, $\left.\mathbf{S}(\mathbf{p}(t))\right|_{-\epsilon<e(t)<\epsilon}$ we can apply only the $\mathbf{K}_{r e f}$ feedback and $\mathbf{G}_{\text {ref }}$ observer gains. Further, $\epsilon$ can be as small which does not cause numerical problems during the calculations. Moreover, based on our investigations a tighter bound (eg. $\epsilon=10^{-6}$ ) does not cause numerical problems. The $\mathbf{K}_{r e f}$ feedback and $\mathbf{G}_{r e f}$ observer gains are the optimal gains for $\mathbf{S}\left(\mathbf{p}_{\text {ref }}\right)$ LTI system. In the small "environment" of $\mathbf{S}\left(\mathbf{p}_{\text {ref }}\right)$, when $\mathbf{S}(\mathbf{p}(t))$ is near to equal $\mathbf{S}\left(\mathbf{p}_{\text {ref }}\right)$, the $\mathbf{K}_{\text {ref }}$ controller is able to handle the LPV system $\left.\mathbf{S}(\mathbf{p}(t))\right|_{-\epsilon<e(t)<\epsilon}$ and $\mathbf{G}_{r e f}$ observer gain can be used. 


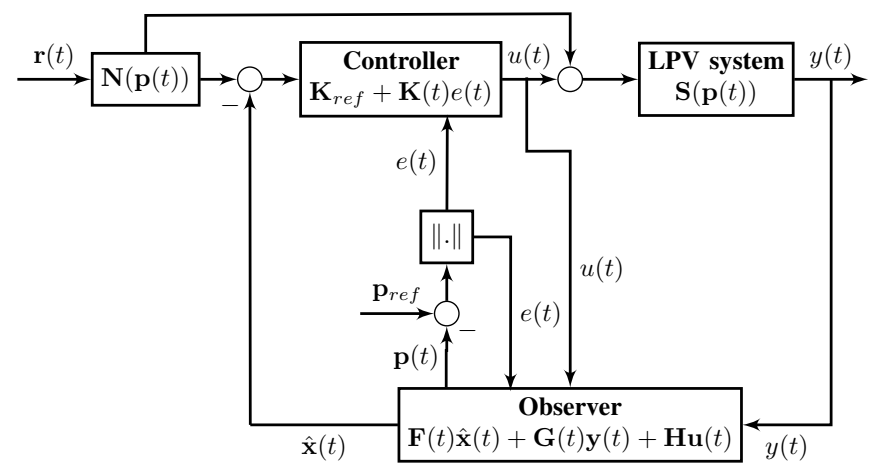

Figure 1: General observer based feedback control loop with completed controller and observer

In classical state feedback control the purpose of the control is to take the states into zero over time. If the control task is to follow given reference signals, a complement is needed. For that purpose the compensator block represented by the compensator matrix $\mathbf{N}=\left[\begin{array}{ll}\mathbf{N}_{x} & \mathbf{N}_{u}\end{array}\right]^{T}$ have to be used [13], [17].

However, in this LPV case the $\mathbf{A}(\mathbf{p}(t))$ is parameter dependent and $\mathbf{p}(t)$ vary in time. Due to this fact, the compensator block has to follow these changes and it should be parameter dependent, namely $\mathbf{N}(\mathbf{p}(t))$. The parameter dependent compensator matrices can be calculated, as follows:

$$
\begin{aligned}
& {\left[\begin{array}{cc}
\mathbf{A}(\mathbf{p}(t)) & \mathbf{B} \\
\mathbf{I}_{n} & \mathbf{0}_{n \times m}
\end{array}\right]\left[\begin{array}{l}
\mathbf{N}_{x} \\
\mathbf{N}_{u}
\end{array}\right]=\left[\begin{array}{c}
\mathbf{0}_{n \times m} \\
\mathbf{I}_{m}
\end{array}\right]} \\
& {\left[\begin{array}{l}
\mathbf{N}_{x} \\
\mathbf{N}_{u}
\end{array}\right]=\left[\begin{array}{cc}
\mathbf{A}(\mathbf{p}(t)) & \mathbf{B} \\
\mathbf{I}_{n} & \mathbf{0}_{n \times m}
\end{array}\right]^{-1}\left[\begin{array}{c}
\mathbf{0}_{n \times m} \\
\mathbf{I}_{m}
\end{array}\right]}
\end{aligned}
$$

where $I_{n}$ is the feedback "selector" matrix (here is a unity matrix), $O_{n \times m}$ is zero matrix and $I_{m}$ is unity matrix.

Finally, the completed LPV controller and observer structure can be seen on Fig. 1. It can be seen that the controller uses the $\hat{\mathbf{x}}(t)$ observed state for controller design, moreover, the $\mathbf{p}(t)$ is constructed by using states of the observer.

\section{CONTROL OF NONLINEAR COMPARTMENTAL SYSTEM}

\section{A. Selected nonlinear compartmental model}

Let an arbitrary compartmental model given by the following equations:

$$
\begin{aligned}
& \dot{x_{1}}(t)= \\
& -k \frac{x_{1}(t)}{1+a x_{1}(t)}+b x_{2}(t)-c\left(x_{2}(t)+z\right) x_{1}(t)+\frac{u_{1}(t)}{V_{1}} \\
& \dot{x_{2}}(t)=-k \frac{x_{2}(t)}{\left(1+d x_{2}(t)\right)}-b x_{2}(t)+\frac{u_{2}(t)}{V_{2}} \\
& y_{1}(t)=x_{1}(t) \\
& y_{2}(t)=x_{2}(t)
\end{aligned}
$$

where $a=0.4[\mathrm{~L} / \mathrm{mmol}], b=0.1[1 / \mathrm{min}], c=0.5[1 / \mathrm{min}]$, $d=0.005[\mathrm{~L} / \mathrm{mmol}], k=0.8[1 / \mathrm{min}], z=0.1[\mathrm{mmol} / \mathrm{L}]$, $V_{1}=2[\mathrm{~L}]$ and $V_{2}=1[\mathrm{~L}]$. The $x_{1}(t)$ and $x_{2}(t)$ are the states and outputs as well. The $u_{1}$ and $u_{2}[\mathrm{mmol} / \mathrm{min}]$ are the inputs. The model has three nonlinearities: the natural degradations of the compartments are loaded with Michaelis-Menten-type saturations and $x_{2}$ has a coupling to an output of $x_{1}$.

The selected scheduling variables were $p=$ $\left[\frac{k}{1+a x_{1}(t)}, x_{2}(t)+z, \frac{k}{1+d x_{2}(t)}\right]^{T}$, which means we have a $3 D$ parameter space.

The state space representation and the state matrices of the LPV system in affine LPV case can be written, as follows:

$$
\begin{gathered}
\left(\begin{array}{l}
\dot{x}_{1}(t) \\
\dot{x}_{2}(t) \\
y_{1}(t) \\
y_{2}(t)
\end{array}\right)=\mathbf{S}(\mathbf{p}(t))\left(\begin{array}{l}
x_{1}(t) \\
x_{2}(t) \\
u_{1}(t) \\
u_{2}(t)
\end{array}\right)=\left[\begin{array}{cc}
\mathbf{A}(\mathbf{p}(t) & \mathbf{B} \\
\mathbf{C} & \mathbf{D}
\end{array}\right]\left(\begin{array}{l}
x_{1}(t) \\
x_{2}(t) \\
u_{1}(t) \\
u_{2}(t)
\end{array}\right) \\
\mathbf{A}(\mathbf{p}(t))=\left[\begin{array}{cc}
0 & b \\
0 & -b
\end{array}\right]+\left[\begin{array}{ll}
-1 & 0 \\
0 & 0
\end{array}\right] p_{1}(t) \\
+\left[\begin{array}{cc}
0 & 0 \\
-c & 0
\end{array}\right] p_{2}(t)+\left[\begin{array}{cc}
0 & 0 \\
0 & -1
\end{array}\right] p_{3}(t) \\
\mathbf{B}=\left[\begin{array}{cc}
1 / V_{1} & 0 \\
0 & 1 / V_{2}
\end{array}\right] \quad \mathbf{C}=\left[\begin{array}{ll}
1 & 0 \\
0 & 1
\end{array}\right] \quad \mathbf{D}=\left[\begin{array}{ll}
0 & 0 \\
0 & 0
\end{array}\right]
\end{gathered}
$$

\section{B. Completed LPV Controller Design}

Consider that the reference parameter vector is $\mathbf{p}_{\text {ref }}=$ $[0.6667,0.6,0.64]^{T}$ (where $\left[x_{1, d}, x_{2, d}\right]^{T}=[0.5,0.5]^{T}$ ). At the reference point, the $\mathbf{A}\left(\mathbf{p}_{\text {ref }}\right)$ is equal to:

$$
\mathbf{A}\left(\mathbf{p}_{\text {ref }}\right)=\left[\begin{array}{cc}
-0.6697 & 0.1 \\
0 & -0.74
\end{array}\right] \text {. }
$$

The eigenvalues of the $\mathbf{A}\left(\mathbf{p}_{\text {ref }}\right)$ are $\lambda=$ $[-0.6697,-0.74]^{T}$, i.e. the reference LTI system is stable, however, the poles are close to zero.

The rank of the controllability matrix was equal to 2 , i.e. the reference LTI system is controllable $(n=2)$ and reference controller design $\mathbf{K}_{r e f}$ is possible.

For that purpose MATLAB ${ }^{\mathrm{TM}}$ care order was used to design the $\mathbf{K}_{r e f}$ gain beside $\mathbf{Q}=I_{2}$ (unity matrix) and $\mathbf{R}=0.01 I_{2}$.

The embedded care order calculates the unique solution for $\mathbf{X}$ in continuous-time control algebraic Ricatti equation [18]:

$$
\begin{aligned}
& \mathbf{A}^{T} \mathbf{X} \mathbf{E}+\mathbf{E}^{T} \mathbf{X A} \\
& \quad-\left(\mathbf{E}^{T} \mathbf{X B}+\mathbf{S}\right) \mathbf{R}^{-1}\left(\mathbf{B}^{T} \mathbf{X E}+\mathbf{S}^{T}\right)+\mathbf{Q}=\mathbf{O}
\end{aligned}
$$

and returns with an optimal gain $\mathbf{G}=\mathbf{R}^{-1}\left(\mathbf{B}^{T} \mathbf{X E}+\mathbf{S}^{T}\right)$. The applied the following parameters: $\mathbf{Q}=\mathbf{I}_{2}, \mathbf{R}=0.01 \mathbf{I}_{2}$, $\mathbf{S}=\mathbf{0}$ and $\mathbf{E}=\mathbf{I}$.

The calculated optimal gain was the following:

$$
\mathbf{K}_{\text {ref }}=\left[\begin{array}{cc}
8.7493 & 0.058 \\
0.1161 & 9.2883
\end{array}\right]
$$

This $\mathbf{K}_{r e f}$ provides that the eigenvalues of the closed-loop reference state matrix $\mathbf{A}\left(\mathbf{p}_{\text {ref }}\right)-\mathbf{B} \mathbf{K}_{\text {ref }}$ become $\lambda_{\text {ref,closed }}=$ 
$[-5.046,-10.0267]^{T}$ - which is a good improvement, since, the eigenvalues are much far from zero without any imaginary component.

The completed controller structure will provides that the eigenvalues of the parameter dependent LPV system's closedloop state matrix $\lambda_{L P V \text {,closed }}$ will be equal to $\lambda_{\text {ref,closed }}$ regardless from the actual value of $\mathbf{p}(t)$. From here, $\mathbf{K}(t)$ can be calculated at each iterations as $(15)$.

Let the reference signal $\mathbf{r}=[8,7]^{T}$ and the control task is $\mathbf{x}_{\infty}=\mathbf{r}$. In order to reach this criteria, parameter dependent compensator matrix $\mathbf{N}(\mathbf{p}(t))$ has to be used. $\mathbf{N}(\mathbf{p}(t))$ can be calculated based on (17) in every iterations during operation.

The initial states of the LPV system was $\mathbf{x}_{0}=[20,10]^{T}$ and the same initial vector $\hat{\mathbf{x}}_{0}=[20,10]^{T}$ was considered in case of the observer, as well.

The selected bound in order to avoid singularity was $\epsilon=$ $10^{-5}$ during calculation of $\mathbf{K}(t)$ and $\mathbf{G}(t)$ based on (15) and (16).

\section{Completed LPV Observer Design}

If, we consider that the state variable of the LPV system are not measurable during operation, a state observer should be used. Moreover, the "state based" scheduling variables - which are used by the controller - are provided by the observer.

The rank of the observability matrix was 2 , namely, the reference LTI system was observable.

We have designed the reference observer gain $\mathbf{G}_{r e f}$ by using the MATLAB ${ }^{\mathrm{TM}}$ place order [18]. The occurred $\mathbf{G}_{r e f}$ was the following:

$$
\mathbf{G}_{\text {ref }}=\left[\begin{array}{cc}
110.47 & -0.3041 \\
-1.3758 & 207.3741
\end{array}\right] \text {. }
$$

Afterwards, the realization of the completed observer structure is possible (as in Fig. 1).

\section{Results}

In order to reach realistic results - since the selected nonlinear compartmental model was physiological one - we have applied lower bound saturation on the control signal $u_{\text {min }}=0 \forall t$ in every case during operation. This complement does not allow the occurrence of physiological not relevant (negative) control inputs.

We compared the controlled LPV system (without observer) to the controlled and observed LPV system.

In the first case only completed LPV controller was used without observer. The results can be seen on Fig. 2. The upper diagram shows the changing of the state variables of the controlled LPV system, while the lower left diagram shows the outputs of the controlled LPV system. It can be seen that the completed LPV controller works well - the states (and outputs) reached the reference over short time and the controller was able to keep them on the given level.
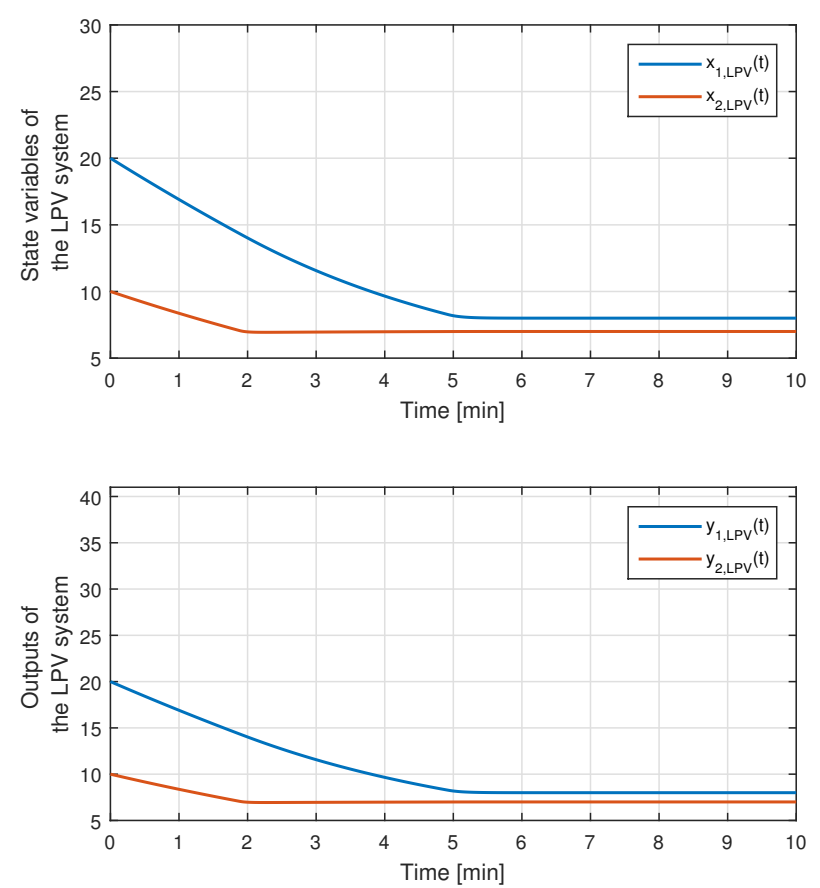

Figure 2: States and outputs of the controlled LPV and controlled and observed LPV system

In the second case the completed LPV controller and observer structure was used. The upper diagram shows the changing of the estimated state variables provided by the completed observer. The lower right diagram represents the output of the controlled and observed LPV system.
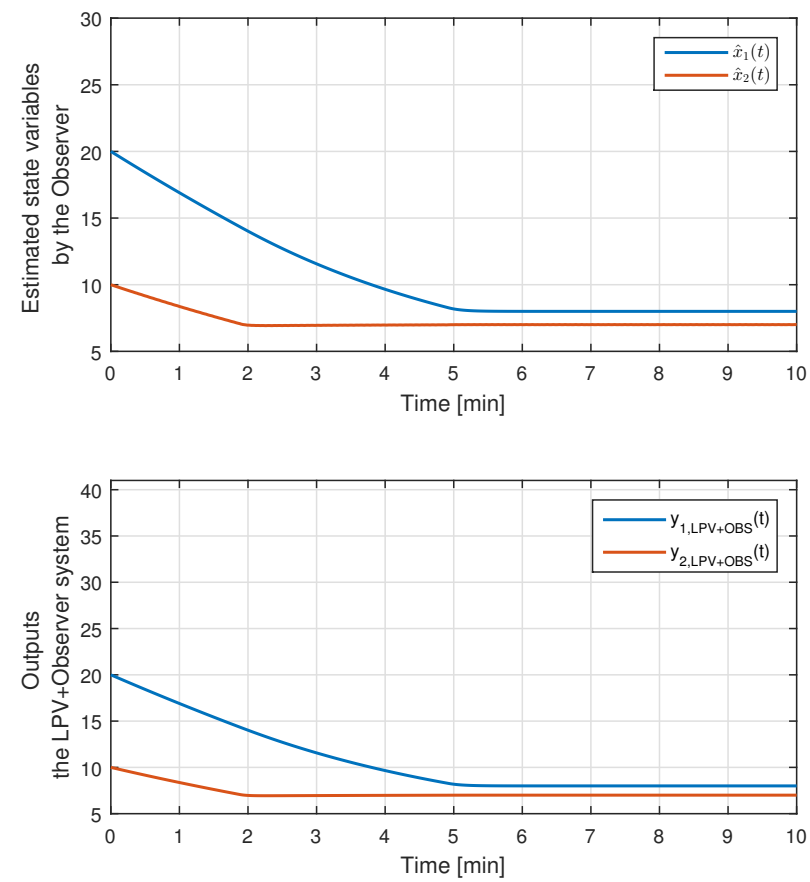

Figure 3: States and outputs of the controlled LPV and controlled and observed LPV system 
It can be seen, that both systems reach their desired steady state values without static error $\mathbf{x}_{\infty}=\mathbf{r}=[8,7]^{T}$. It looks, there is no difference between the outputs and states of the given LPV systems.

In order to estimate the exact error between the controlled LPV and the controlled and observed LPV systems we compared the numeric values of the simulated system in given time instances.

As Fig. 4. shows, a small, oscillating error occurred between the states and outputs of the given systems. However, the order of the error was around $10^{-3}-10^{-4}$, which means only small deviation and numerical error occurred.

Fig. 5. shows the PS of the simple LPV system and the PS, which is realized by the observer. The order of the error between the scheduling variables are very low: $10^{-2}$, which means the completed observer approximates the scheduling variables, however, with high accuracy.

In both cases the varying LPV systems did not get close to the reference system, namely, the trajectory of the $\mathbf{p}(t)$ (blue line on the upper diagram of Fig. 5) was not closing to $\mathbf{p}_{\text {ref }}$ (red cross on the upper diagram of Fig. 5) during operation. The $e(t)=\left\|\mathbf{p}_{\text {ref }}-\mathbf{p}(t)\right\|_{2}=0$ did not become during operation.

\section{CONCLUSION}

In this study we have introduced a novel "completed" LPV controller and observer design approach for control of nonlinear systems. The developed method is a mixture of classical state feedback theorems and a supplementary controller and observer structure which exploit the special properties of the parameter space of the LPV system. The main benefit of the proposed method is that the controller design does not require highly advanced mathematical tools and high computational capacity - the basis is the classical state feedback design in case of LTI systems. Through the introduced framework the developed completed LPV controller enforces the given LPV system and via the given nonlinear system to behave as a selected linear LTI system. In other words, the nonlinear system to be controlled via LPV framework will mimics the behavior of a selected and controlled LTI system.

We have demonstrated the usability of our method in case of a highly nonlinear compartmental model. The results have shown that the completed LPV controller is able to handle the system with good performance.

In our future work we are going to investigate how can be the proposed method extended to general cases, for example in case of non-affine nonlinear systems.

\section{ACKNOWLEDGMENT}

Gy. Eigner and P. Pausits thankfully acknowledge the support of the Robotics Special College and the Doctoral School of Applied Informatics and Applied Mathematics of Obuda University. The research was also supported by the Research and Innovation Center of Obuda University and the European Research Council Starting Grant ERC-StG 679681.
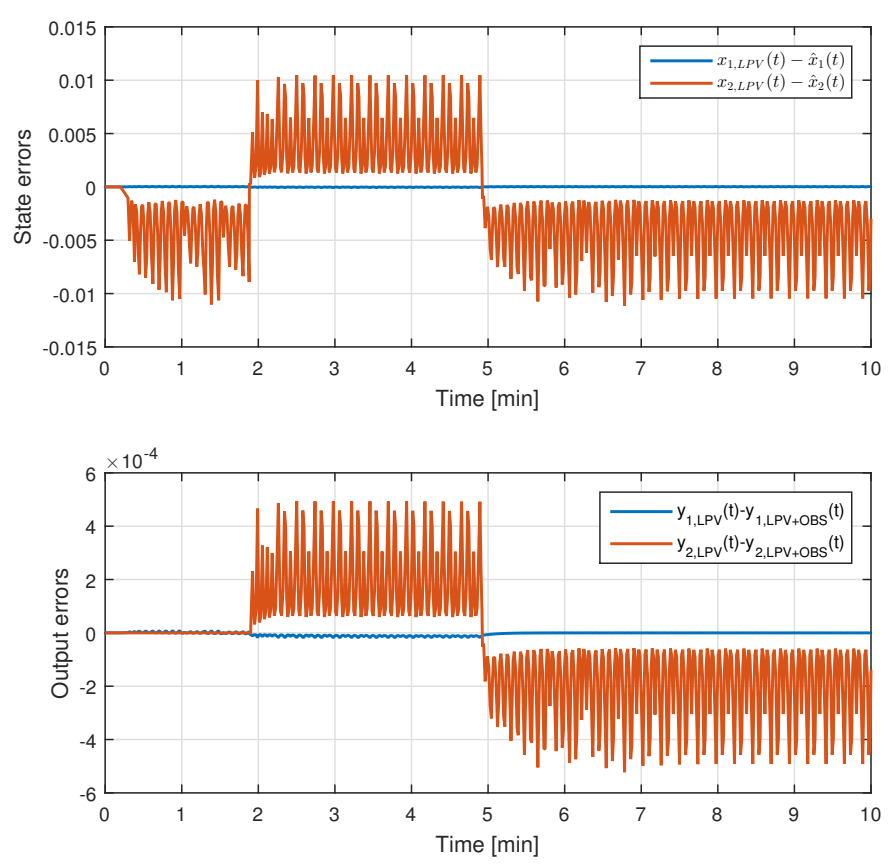

Figure 4: Result of the simulations
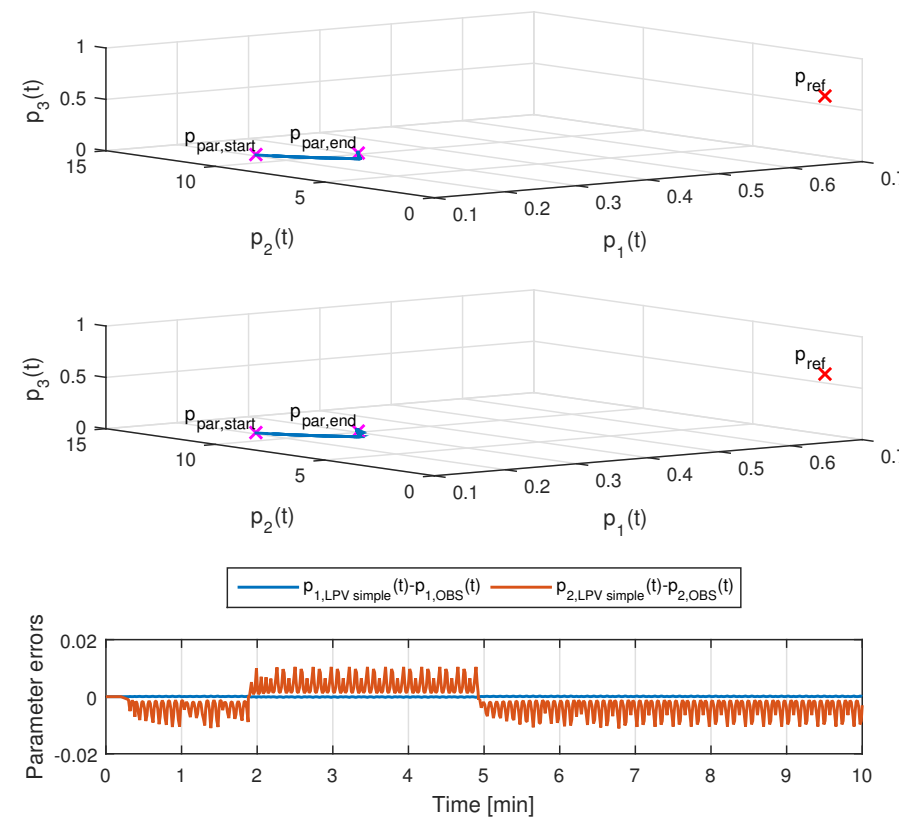

Figure 5: Parameter space and parameter errors. Upper row: PS of LPV system; middle row: PS of the observer; lower row: parameter error

\section{REFERENCES}

[1] J. Bronzino and D. Peterson, Eds., The Biomedical Engineering Handbook, 4th ed. Boca Raton, USA: CRC Press, 2016.

[2] A. Lyapunov, "A general task about the stability of motion (in russian)," Ph.D. dissertation, University of Kharkov, Kharokov, Russia, 1892.

[3] - Stability of Motion. New York, USA: Academic Press, 1966.

[4] J.K. Tar, J.F. Bitó, L. Nádai, and J.A.T. Machado, "Robust Fixed Point Transformations in Adaptive Control Using Local Basin of Attraction," ACTA Pol Hung, vol. 6, no. 1, pp. 21-37, 2009. 
[5] J. Tar, L. Nadai, and I. Rudas, Eds., System and Control Theory with Especial Emphasis on Nonlinear Systems, 1st ed. Budapest, Hungary: Typotex, 2012.

[6] A. White, G. Zhu, and J. Choi, Linear Parameter Varying Control for Engineering Applicaitons, 1st ed. London: Springer, 2013.

[7] Gy. Eigner, J.K. Tar, I. Rudas, and L. Kovacs, "LPV-based quality interpretations on modeling and control of diabetes," ACTA Pol Hung, vol. 13, no. 1, pp. 171 - 190, 2016.

[8] F. Wettl, Linear Algebra [in Hungarian], 1st ed. Budapest, Hungary: Budapest University of Technology and Economy, Faculty of Natural Sciences, 2011.

[9] C. Scherer and S. Weiland, "Lecture Notes DISC Course on Linear Matrix Inequalities in Control," Delft University, 1999.

[10] O. Sename, P. Gáspár, and J. Bokor, "Robust control and linear parameter varying approaches, application to vehicle dynamics," ser. Lecture Notes in Control and Information Sciences. Berlin: Springer-Verlag, 2013, vol. 437.
[11] R. Burns, Ed., Advanced Control Engineering, 1st ed. Oxford, UK: Butterworth-Heinemann, 2001.

[12] W. Levine, The Control Engineering Handbook, 2nd ed. Boca Raton: CRC Press, Taylor and Francis Group, 2011.

[13] B. Lantos, Theory and design of control systems [in Hungarian], 2nd ed. Budapest, Hungary: Akademia Press, 2005.

[14] R. Beezer, A First Course in Linear Algebra, version 3.40 ed. Washington, USA: Congruent Press, 2014.

[15] G. Eigner and L. Kovacs, "LPV-based controller design for physiological systems: A novel approach,” ACTA Polytechnica Hungarica, Manuscript. Submitted in 2016.

[16] G. Eigner, "Working Title: Closed-Loop Control of Physiological Systems," Ph.D. dissertation, Applied Informatics and Applied Mathemathics Doctoral School, Óbuda University, Budapest, Hungary, Manuscript. Planned defense: 2016.

[17] D. Leith and W. Leithead, "Survey of gain-scheduling analysis design," International Journal of Control, vol. 73, pp. 1001-1025, 1999.

[18] Control System Toolbox Getting Started Guide. 